\title{
A tool for symmetry breaking and multiplicity in some nonlocal problems
}

\author{
Roberta Musina * and Alexander I. Nazarov ${ }^{* *}$
}

\begin{abstract}
We prove some basic inequalities relating the Gagliardo-Nirenberg seminorms of a symmetric function $u$ on $\mathbb{R}^{n}$ and of its perturbation $u \varphi_{\mu}$, where $\varphi_{\mu}$ is a suitably chosen eigenfunction of the Laplace-Beltrami operator on the sphere $\mathbb{S}^{n-1}$, thus providing a technical but rather powerful tool to detect symmetry breaking and multiplicity phenomena in variational equations driven by the fractional Laplace operator. A concrete application to a problem related to the fractional Caffarelli-Kohn-Nirenberg inequality is given.
\end{abstract}

Keywords: Fractional Laplacian, Symmetry breaking, Multiplicity

2010 Mathematics Subject Classfication: 35R11; 35B06; 35A02.

\section{Introduction}

Let $\varphi_{\mu}$ be an eigenfunction of the Laplace-Beltrami operator on the sphere $\mathbb{S}^{n-1}, n \geq 2$, relative to the positive eigenvalue $\mu$ and normalized by the condition

$$
f_{\mathbb{S}^{n-1}} \varphi_{\mu}^{2} d \sigma=1 .
$$

Take a "good" radial function $u$ on $\mathbb{R}^{n}$. We break the symmetry of $u$ by defining, via polar coordinates, $\left(u \varphi_{\mu}\right)(r \sigma)=u(r) \varphi_{\mu}(\sigma)$. The function $u \varphi_{\mu}$ has the same $L^{2}$ norm as $u$ and it is orthogonal to $u$ in $L^{2}$. A popular and efficient technique to detect symmetry breaking

\footnotetext{
*Dipartimento di Matematica ed Informatica, Università di Udine, via delle Scienze, 206 - 33100 Udine, Italy. Email: roberta.musina@uniud.it.

** St.Petersburg Dept of Steklov Institute, Fontanka 27, St.Petersburg, 191023, Russia, and St.Petersburg State University, Universitetskii pr. 28, St.Petersburg, 198504, Russia. E-mail: al.il.nazarov@gmail.com.
} 
and multiplicity results for a large class of variational problems is based on the comparison between the energies of $u$ and $u \varphi_{\mu}$. We cite for instance [26, 13, where the trivial equality

$$
\int_{\mathbb{R}^{n}}\left|\nabla\left(u \varphi_{\mu}\right)\right|^{2} d x-\int_{\mathbb{R}^{n}}|\nabla u|^{2} d x=\mu \int_{\mathbb{R}^{n}}|x|^{-2}|u|^{2} d x
$$

is crucially used to tackle certain problems driven by the Laplace operator $-\Delta$. We cite also [23, 27, 17, 18, 5, 24, where the $p$-Laplacian or more general second order, possibly degenerate operators in divergence form are considered, and [1, 4], that deal with fourthorder variational equations.

In dealing with variational problems involving the Dirichlet Laplacian $(-\Delta)^{s}, 0<s<1$, a simple and powerful identity comparable with (1.2) is hopeless. However, as a corollary of the more general Lemma 3.1 in Section 3, we obtain the existence of a positive constant $c_{\mu}$, not depending on $u$, such that

$$
\int_{\mathbb{R}^{n}}\left|(-\Delta)^{\frac{s}{2}}\left(u \varphi_{\mu}\right)\right|^{2} d x-\int_{\mathbb{R}^{n}}\left|(-\Delta)^{\frac{s}{2}} u\right|^{2} d x \leq c_{\mu} \int_{\mathbb{R}^{n}}|x|^{-2 s}|u|^{2} d x .
$$

Besides its impact on the study of fractional differential equations, inequality (1.3) might have an independent interest. For instance, it is strongly related to Bochner's relations and to the results in [10, 14].

Inequality (1.3) and its generalizations below provide quite useful technical tools. In order to illustrate their applications in concrete problems we take as model the fractional Hardy-Sobolev inequality

$$
\int_{\mathbb{R}^{n}}\left|(-\Delta)^{\frac{s}{2}} u\right|^{2} d x+\lambda \int_{\mathbb{R}^{n}}|x|^{-2 s}|u|^{2} d x \geq \mathcal{S}_{q}^{\lambda}\left(\int_{\mathbb{R}^{n}}|x|^{-b q}|u|^{q} d x\right)^{\frac{2}{q}}, \quad u \in \mathcal{D}^{s}\left(\mathbb{R}^{n}\right)
$$

and its associated semilinear Euler-Lagrange equation

$$
(-\Delta)^{s} u+\lambda|x|^{-2 s} u=|x|^{-b q} u^{q-1}, \quad u \in \mathcal{D}^{s}\left(\mathbb{R}^{n}\right) .
$$

Inequality (1.4) is clearly related to the fractional Hardy and Sobolev inequalities,

$$
\int_{\mathbb{R}^{n}}\left|(-\Delta)^{\frac{s}{2}} u\right|^{2} d x \geq H_{s} \int_{\mathbb{R}^{n}}|x|^{-2 s}|u|^{2} d x, \int_{\mathbb{R}^{n}}\left|(-\Delta)^{\frac{s}{2}} u\right|^{2} d x \geq S_{2_{s}^{*}}\left(\int_{\mathbb{R}^{n}}|u|^{2_{s}^{*}} d x\right)^{\frac{2}{2_{s}^{*}}}
$$

where $2_{s}^{*}=\frac{2 n}{n-2 s}$ is the critical Sobolev exponent. The explicit values of the Hardy constant $H_{s}$ and of the Sobolev constant $S_{2_{s}^{*}}$ have been computed in [15], 6], respectively. 
From now on, we take exponents $q, b$ satisfying

$$
2<q<2_{s}^{*}=\frac{2 n}{n-2 s}, \quad \frac{n}{q}-b=\frac{n}{2}-s .
$$

By using Hölder's interpolation, it is easy to see that (1.4) holds with a positive best constant $S_{q}^{\lambda}$ provided that $\lambda>-H_{s}$. Under these assumptions, nowadays standard arguments give the existence of an extremal for the noncompact minimization problem

$$
S_{q}^{\lambda}=\inf _{\substack{u \in \mathcal{D}^{s}\left(\mathbb{R}^{n}\right) \\ u \neq 0}} J_{\lambda}(u), \quad J_{\lambda}(u):=\frac{\left\|(-\Delta)^{\frac{s}{2}} u\right\|_{2}^{2}+\lambda\left\||x|^{-s} u\right\|_{2}^{2}}{\left\||x|^{-b} u\right\|_{q}^{2}},
$$

see Corollary 4.3 in Section 4. Thanks to (1.3), in Section 5 we prove the next result.

Theorem 1.1 If $\lambda>0$ is large enough then symmetry breaking occurs, that is, no extremal for $S_{q}^{\lambda}$ is radially symmetric.

Up to a Lagrange multiplier, any extremal $\underline{u}^{\lambda}$ for $S_{q}^{\lambda}$ is nonnegative and solves (1.5) in the weak sense. By Theorem 1.1 and Lemma 4.2 in Section 4 , we have that for $\lambda$ large enough, problem (1.5) has in fact two distinct nonnegative solutions: the ground state solution $\underline{u}^{\lambda}$, which is not radial, and a radial one, that minimizes $J_{\lambda}(u)$ on the space of radial functions.

The exploitation of different symmetries and Lemma 3.1 lead to the next multiplicity results.

Theorem 1.2 Let $n \geq 2$ be even. For any integer $h \geq 1$, there exists $\lambda_{h}>0$ such that for $\lambda>\lambda_{h}$, problem (1.5) has at least $h$ nonnegative solutions, that are distinct modulo rotations of $\mathbb{R}^{n}$.

Theorem 1.3 Let $n \geq 3$ be odd. Then for $\lambda$ large enough problem (1.5) has $N(n) \geq 4$ nonnegative solutions, that are distinct modulo rotations of $\mathbb{R}^{n}$.

Let us conclude this introduction by pointing out few facts.

If $\lambda \in\left(-H_{s}, 0\right]$, then any nonnegative solution to (1.5) is radially symmetric about the origin; for the proof, notice that $b>0$ and adapt the moving plane argument in [7]. In particular, any extremal for $S_{q}^{\lambda}$ is radially symmetric if $\lambda \leq 0$ (the last statement can be also proved by the Schwarz symmetrization, see, e.g., [16, Theorem 2.31 and Sec. II.2]).

By Theorem 1.1, there exists an optimal parameter $\widehat{\lambda}=\widehat{\lambda}(n, s, q) \geq 0$ such that symmetry breaking occurs whenever $\lambda>\hat{\lambda}$. In the local case $s=1$, symmetry breaking has been firstly pointed out in [3] 1 Nowadays the picture is complete, thanks to the results in

\footnotetext{
${ }^{1}$ The equation considered in [3] is related to (1.5) with $s=1$ via functional change $u(x) \mapsto|x|^{a} u(x)$.
} 
[11, 9, 8], one gets that $\widehat{\lambda}=\frac{4(n-1)}{q^{2}-4}$.

In the nonlocal case we cannot prove even whether the set of $\lambda$ providing the symmetry breaking is connected, nor we have any conjecture about the value of $\hat{\lambda}$. By exploiting our proof and thanks to Remark 2.2, one can find rough upper bounds on $\widehat{\lambda}$ in case $n \geq 4$, or $n=3$ and $0<s \leq \frac{1}{2}$.

The paper is organized as follows. In the next two sections we prove some crucial inequalities, including (1.3). The main tools are the Caffarelli-Silvestre extension technique [2] and the results in 22]. Section 4 contains the main variational tools and a criterion to distinguish solutions to (1.5) enjoing different symmetry properties, see the Basic Lemma 4.5. The proofs of the main Theorems are collected in Section 5 .

Remark 1.4 Minor modifications in the variational arguments give simmetry breaking and multiplicity of positive solutions to the Dirichlet problem

$$
\begin{cases}(-\Delta)^{s} u=u^{q-1} & \text { in } A=\{R<|x|<R+1\} \subset \mathbb{R}^{n} \\ u=0 & \text { in } \mathbb{R}^{n} \backslash A\end{cases}
$$

for $q \in\left(2,2_{s}^{*}\right)$ and $R$ large. See however [28], where a different argument is used.

\section{Preliminaries}

The fractional Laplacian $(-\Delta)^{s}$ in $\mathbb{R}^{n}, n \geq 2$, is formally defined by

$$
\mathcal{F}\left[(-\Delta)^{s} u\right]=|\xi|^{2 s} \mathcal{F}[u],
$$

where $\mathcal{F}=\mathcal{F}[u](\xi)=(2 \pi)^{-\frac{n}{2}} \int_{\mathbb{R}^{n}} e^{-i \xi \cdot x} u(x) d x$ is the Fourier transform.

Thanks to the Sobolev inequality, the space

$$
\mathcal{D}^{s}\left(\mathbb{R}^{n}\right)=\left\{u \in L^{2_{s}^{*}}\left(\mathbb{R}^{n}\right) \mid(-\Delta)^{\frac{s}{2}} u \in L^{2}\left(\mathbb{R}^{n}\right)\right\}
$$

naturally inherits a Hilbertian structure from the scalar product

$$
(u, v)=\int_{\mathbb{R}^{n}}(-\Delta)^{\frac{s}{2}} u(-\Delta)^{\frac{s}{2}} v d x=\int_{\mathbb{R}^{n}}|\xi|^{2 s} \mathcal{F}[u] \overline{\mathcal{F}[v]} d \xi .
$$

From now on, we will always use the shorter notation $\mathcal{D}^{s}$ instead of $\mathcal{D}^{s}\left(\mathbb{R}^{n}\right)$. 
In the breakthrough paper [2], Caffarelli and Silvestre investigated the relations between the nonlocal operator $(-\Delta)^{s}$ in $\mathbb{R}^{n} \ni x$ and the pointwise defined differential operator $-\operatorname{div}\left(y^{1-2 s} \nabla\right)$ in $\mathbb{R}_{+}^{n+1} \equiv \mathbb{R}^{n} \times(0, \infty) \ni(x, y)$. It turns out that any function $w$ in the space

$$
\mathcal{W}^{s}=\mathcal{W}^{s}\left(\mathbb{R}_{+}^{n+1}\right)=\left\{w: \mathbb{R}_{+}^{n+1} \rightarrow \mathbb{R} \text { measurable, } \int_{0}^{\infty} \int_{\mathbb{R}^{n}} y^{1-2 s}|\nabla w|^{2} d x d y<\infty\right\}
$$

has a trace on the boundary of $\mathbb{R}_{+}^{n+1},\left.w\right|_{\partial \mathbb{R}_{+}^{n+1}} \in \mathcal{D}^{s}$ and for any $u \in \mathcal{D}^{s}$ we have that

$$
\int_{\mathbb{R}^{n}}\left|(-\Delta)^{\frac{s}{2}} u\right|^{2} d x=\inf _{\substack{w \in \mathcal{W}^{s} \\ w \mid \\ \partial \mathbb{R}_{+}^{n+1}=u}} C_{s} \int_{0}^{\infty} \int_{\mathbb{R}^{n}} y^{1-2 s}|\nabla w|^{2} d x d y, \quad C_{s}=\frac{\Gamma(s)}{2^{1-2 s} \Gamma(1-s)} .
$$

The Caffarelli-Silvestre extension $w_{u}$ of a function $u \in \mathcal{D}^{s}$ is the unique solution to the convex minimization problem in (2.1), hence it satisfies

$$
\begin{gathered}
-\operatorname{div}\left(y^{1-2 s} \nabla w_{u}\right)=0 \quad \text { in } \mathbb{R}_{+}^{n+1}, \quad w_{u}(x, 0)=u(x), \\
C_{s} \int_{0}^{\infty} \int_{\mathbb{R}^{n}} y^{1-2 s}\left|\nabla w_{u}\right|^{2} d x d y=\int_{\mathbb{R}^{n}}\left|(-\Delta)^{\frac{s}{2}} u\right|^{2} d x .
\end{gathered}
$$

Recall that the Hardy type inequality

$$
\int_{0}^{\infty} \int_{\mathbb{R}^{n}} y^{1-2 s}|\nabla w|^{2} d x d y \geq\left(\frac{n-2 s}{2}\right)^{2} \int_{0}^{\infty} \int_{\mathbb{R}^{n}} y^{1-2 s} \frac{|w|^{2}}{|x|^{2}+y^{2}} d x d y, \quad w \in \mathcal{W}^{s}
$$

holds with a sharp and not achieved constant, see [20, Section 2]. In particular, $\mathcal{W}^{s}$ inherits a natural Hilbert space structure and the map $u \mapsto w_{u}$ is an isometry, up to the constant $C_{s}$. In the next lemma we provide a crucial relation between the Hardy integrals of $u \in \mathcal{D}^{s}$ and of its extension $w_{u} \in \mathcal{W}^{s}$.

Lemma 2.1 Let $w_{u}$ be the Caffarelli-Silvestre extension of $u \in \mathcal{D}^{s}$. Then

$$
\int_{0}^{\infty} \int_{\mathbb{R}^{n}} y^{1-2 s} \frac{\left|w_{u}\right|^{2}}{|x|^{2}+y^{2}} d x d y \leq \gamma \int_{\mathbb{R}^{n}} \frac{|u|^{2}}{|x|^{2 s}} d x
$$

where the positive constant $\gamma$ does not depend on $u$. 
Proof. By [22, Theorem 1] there exists a constant $\hat{c}>0$, possibly depending on $n, s$ but not on $u$, such that

$$
\int_{\mathbb{R}^{n}} \frac{\left|w_{u}(x, y)\right|^{2}}{|x|^{2}+y^{2}} d x \leq \hat{c} \int_{\mathbb{R}^{n}} \frac{|u(x)|^{2}}{|x|^{2}+y^{2}} d x \quad \text { for any } y>0
$$

Since

$$
\int_{0}^{\infty} \int_{\mathbb{R}^{n}} y^{1-2 s} \frac{|u(x)|^{2}}{|x|^{2}+y^{2}} d x d y=\int_{\mathbb{R}^{n}}|u(x)|^{2} d x \int_{0}^{\infty} \frac{y^{1-2 s}}{|x|^{2}+y^{2}} d y=\frac{1}{2} \Gamma(s) \Gamma(1-s) \int_{\mathbb{R}^{n}} \frac{|u(x)|^{2}}{|x|^{2 s}} d x,
$$

the conclusion follows immediately, with $\gamma=\hat{c} \Gamma(s) \Gamma(1-s) / 2$.

Remark 2.2 By [22, Theorem 2] we know that [2.4) holds with $\hat{c}=1$, provided that $n \geq 4$ or $n=3$ and $0<s \leq \frac{1}{2}$. In this case we obtain the estimate

$$
\int_{0}^{\infty} \int_{\mathbb{R}^{n}} y^{1-2 s} \frac{\left|w_{u}\right|^{2}}{|x|^{2}+y^{2}} d x d y \leq \frac{\pi}{2 \sin (\pi s)} \int_{\mathbb{R}^{n}} \frac{|u|^{2}}{|x|^{2 s}} d x \quad \text { for any } u \in \mathcal{D}^{s} .
$$

We conjecture that (2.5) holds with a sharp constant, at least for $n \geq 3$. The lowest dimensional case $n=2$ looks more obscure. Finally, it would be of interest to investigate whether (2.3) holds in case $n=1, s \in\left(0, \frac{1}{2}\right)$.

\section{Perturbing symmetric functions}

Let $n=k m$ with $k \geq 2, m \geq 1$, and write $\mathbb{R}^{n}$ as the Cartesian product of $m$ copies of $\mathbb{R}^{k}$. It is convenient to denote by $\mathbb{R}_{j}^{k}$ the $j$-th copy of $\mathbb{R}^{k}$, so that $\mathbb{R}^{n}=\mathbb{R}_{1}^{k} \times \cdots \times \mathbb{R}_{m}^{k}$. The variable in $\mathbb{R}_{j}^{k}$ is $x_{j}$; its polar coordinates are $r_{j}=\left|x_{j}\right|, \sigma_{j} \in \mathbb{S}_{j}^{k-1}$, where $\mathbb{S}_{j}^{k-1}$ is the unit sphere in $\mathbb{R}_{j}^{k}$.

In the next crucial lemma we take a proper closed subgroup $\mathcal{G}_{k}$ of $O(k)$ and an eigenfunction $\phi$ for the Laplace-Beltrami operator on $\mathbb{S}^{k-1}$, solving

$$
\left\{\begin{array}{l}
-\Delta_{\sigma} \phi=\mu \phi \\
\phi \in H_{\mathcal{G}_{k}}^{1}\left(\mathbb{S}^{k-1}\right)
\end{array} \quad, \quad f_{\mathbb{S}^{k-1}}|\phi|^{2} d \sigma=1, \quad f_{\mathbb{S}^{k-1}} \phi d \sigma=0\right.
$$


for some eigenvalue $\mu>0$, where $H_{\mathcal{G}_{k}}^{1}\left(\mathbb{S}^{k-1}\right)$ is the space of $\mathcal{G}_{k}$-invariant functions in $H^{1}\left(\mathbb{S}^{k-1}\right)$. In particular one can take

$$
\mu=\inf \left\{\frac{\int_{\mathbb{S}^{k-1}}\left|\nabla_{\sigma} \varphi\right|^{2} d \sigma}{\int_{\mathbb{S}^{k-1}}|\varphi|^{2} d \sigma}: \varphi \in H_{\mathcal{G}_{k}}^{1}\left(\mathbb{S}^{k-1}\right), \quad f_{\mathbb{S}^{k-1}} \varphi d \sigma=0\right\} .
$$

To shorten notation we put

$$
\phi_{j}(x)=\phi\left(\frac{x_{j}}{\left|x_{j}\right|}\right) \quad \text { for } j=1, \ldots, m, x=\left(x_{1}, \ldots, x_{m}\right) \ni \mathbb{R}_{1}^{k} \times \ldots \mathbb{R}_{m}^{k} .
$$

Lemma 3.1 Let $\mathbb{R}^{n}=\left(\mathbb{R}^{k}\right)^{m}$, with $k \geq 2, m \geq 1$. Assume that $u \in \mathcal{D}^{s}$ is radially symmetric in each variable $x_{j}$, that is, $u\left(x_{1}, \ldots, x_{m}\right)=u\left(\left|x_{1}\right|, \ldots,\left|x_{m}\right|\right)$. The function

$$
\widetilde{u}(x)=u(x) \sum_{j=1}^{m} \frac{\left|x_{j}\right|}{|x|} \phi_{j}(x)
$$

belongs to $\mathcal{D}^{s}$ and satisfies

$$
\begin{gathered}
\int_{\mathbb{R}^{n}}|x|^{-b q}|u|^{q-2} u \widetilde{u} d x=0, \quad \int_{\mathbb{R}^{n}}|x|^{-b q}|u|^{q-2}|\widetilde{u}|^{2} d x=\int_{\mathbb{R}^{n}}|x|^{-b q}|u|^{q} d x, \\
\int_{\mathbb{R}^{n}}\left|(-\Delta)^{\frac{s}{2}} \widetilde{u}\right|^{2} d x-\int_{\mathbb{R}^{n}}\left|(-\Delta)^{\frac{s}{2}} u\right|^{2} d x \leq c_{\mu} \int_{\mathbb{R}^{n}}|x|^{-2 s}|u|^{2} d x,
\end{gathered}
$$

where $q \in\left[2,2_{s}^{*}\right], b=\frac{n}{q}-\frac{n}{2}+s$ and the constant $c_{\mu}$ does not depend on $u$.

Proof. We start by pointing out the orthogonality relation

$$
\int_{\mathbb{R}^{n}}\left(\sum_{j, h=1}^{m} f_{j} g_{h} \phi_{j} \phi_{h}\right) V d x=\int_{\mathbb{R}^{n}}\left(\sum_{j=1}^{m} f_{j} g_{j}\right) V d x
$$

that holds for functions $V, f_{j}, g_{h}$, each of them satisfying suitable summability assumptions and being radially symmetric in each variable $x_{j}, j=1, \ldots, m$. To prove (3.5) we first notice that

$$
\sum_{h=1}^{m} f_{j} g_{h} f_{\mathbb{S}_{j}^{k-1}} \phi_{j} \phi_{h} d \sigma_{j}=\sum_{h=1}^{m} f_{j} g_{h} \delta_{j h}=f_{j} g_{j} \quad \text { for any } j=1, \ldots, m,
$$


compare with (3.1). Since $V f_{j} g_{h}$ is radially symmetric in $x_{j}$, we infer that

$$
\sum_{h=1}^{m} \int_{\mathbb{R}^{n}} V f_{j} g_{h} \phi_{j} \phi_{h} d x=\int_{\mathbb{R}^{n}} V f_{j} g_{j} d x
$$

so that (3.5) follows by taking the sum for $j=1, \ldots m$.

We are now in position to prove the lemma. The first equality in (3.3) is immediate, because for any index $j=1, \ldots, m$ the function $\phi_{j}=\phi\left(\sigma_{j}\right)$ has null mean on $\mathbb{S}_{j}^{k-1}$, while $\left|x_{j}\right|^{-b q-1}|u|^{q}\left|x_{j}\right|$ is radially symmetric in the variable $x_{j} \in \mathbb{R}_{j}^{k}$.

The second equality in (3.3) follows from (3.5). In fact, $\sum_{j}\left|x_{j}\right|^{2}=|x|^{2}$ and thus

$$
\int_{\mathbb{R}^{n}}|x|^{-b q}|u|^{q-2}|\widetilde{u}|^{2} d x=\int_{\mathbb{R}^{n}}|x|^{-b q}|u|^{q}|x|^{-2}\left(\sum_{j, h=1}^{m}\left|x_{j}\right|\left|x_{h}\right| \phi_{j} \phi_{h}\right) d x=\int_{\mathbb{R}^{n}}|x|^{-b q}|u|^{q} d x .
$$

Next, let $w_{u}=w_{u}(x, y)$ be the Caffarelli-Silvestre extension of $u$. Since $w_{u}$ is uniquely determined as the solution of a convex minimization problem, then clearly $w_{u}(x, y)=$ $w_{u}\left(\left|x_{1}\right|, \ldots,\left|x_{m}\right|, y\right)$ for any $y>0$.

We introduce the following extension $\widetilde{w}$ of $\widetilde{u}$,

$$
\widetilde{w}(x, y)=w_{u}(x, y) \sum_{j=1}^{m} \frac{\left|x_{j}\right|}{\sqrt{|x|^{2}+y^{2}}} \phi_{j}(x) .
$$

From now on we simply write $w$ instead of $w_{u}$. It is also convenient to put

$$
\zeta=(x, y) \in \mathbb{R}_{+}^{n+1}, \quad f_{j}(\zeta)=|\zeta|^{-1}\left|x_{j}\right| \text { for } j=1, \ldots, m, \quad F(\zeta)=\sum_{j=1}^{m} f_{j} \phi_{j} .
$$

We claim that

$$
\int_{0}^{\infty} \int_{\mathbb{R}^{n}} y^{1-2 s}\left(|\nabla \widetilde{w}|^{2}-|\nabla w|^{2}\right) d x d y \leq(m \mu+m+1-2 s) \int_{0 \mathbb{R}^{n}}^{\infty} \int^{1-2 s}|\zeta|^{-2}|w|^{2} d x d y .
$$

To prove (3.6) we use (3.5), (2.4) and notice that $\sum_{j} f_{j}^{2}=|\zeta|^{-2}|x|^{2} \leq 1$ to get

$$
\begin{gathered}
\int_{\mathbb{R}^{n}} y^{1-2 s} F^{2}|\nabla w|^{2} d x \leq \int_{\mathbb{R}^{n}} y^{1-2 s}|\nabla w|^{2} d x \\
-\int_{\mathbb{R}^{n}} F \partial_{y} F|w|^{2} d x=y \int_{\mathbb{R}^{n}}|w|^{2}|\zeta|^{-4}|x|^{2} d x \leq \hat{c} y \int_{\mathbb{R}^{n}}|u|^{2}|\zeta|^{-2} d x .
\end{gathered}
$$


Since $\widetilde{w}=F w$, from (3.7) we easily infer

$$
\int_{0}^{\infty} \int_{\mathbb{R}^{n}} y^{1-2 s}|\nabla \widetilde{w}|^{2} d x d y \leq \int_{0}^{\infty} \int_{\mathbb{R}^{n}} y^{1-2 s}|\nabla w|^{2} d x d y+\int_{0}^{\infty} \int_{\mathbb{R}^{n}} y^{1-2 s}\left(|w|^{2}|\nabla F|^{2}+F \nabla F \cdot \nabla|w|^{2}\right) d x d y .
$$

Since (3.8) and the Lebesgue dominated convergence theorem give

$$
\begin{aligned}
& \left.\lim _{y \rightarrow 0^{+}}\left|\int_{\mathbb{R}^{n}} y^{1-2 s} F \partial_{y} F\right| w\right|^{2} d x \mid \leq \hat{c} \lim _{y \rightarrow 0^{+}} \int_{\mathbb{R}^{n}} y^{2-2 s} \frac{|u|^{2}}{|x|^{2}+y^{2}} d x=0 \\
& \left.\lim _{y \rightarrow \infty}\left|\int_{\mathbb{R}^{n}} y^{1-2 s} F \partial_{y} F\right| w\right|^{2} d x \mid \leq \hat{c} \lim _{y \rightarrow \infty} \int_{\mathbb{R}^{n}} y^{2-2 s} \frac{|u|^{2}}{|x|^{2}+y^{2}} d x=0,
\end{aligned}
$$

(the summable majorant is $\frac{|u|^{2}}{|x|^{2 s}}$ ), we can integrate by parts on $\mathbb{R}_{+}^{n+1}$ to obtain

$$
\begin{aligned}
\int_{0 \mathbb{R}^{n}}^{\infty} y^{1-2 s}\left(|\nabla \widetilde{w}|^{2}-|\nabla w|^{2}\right) d x d y & \leq \int_{0}^{\infty} \int_{\mathbb{R}^{n}}|w|^{2}\left(y^{1-2 s}|\nabla F|^{2}-\operatorname{div}\left(y^{1-2 s} F \nabla F\right)\right) d x d y \\
& =-\int_{0}^{\infty} \int_{\mathbb{R}^{n}}|w|^{2} F \operatorname{div}\left(y^{1-2 s} \nabla F\right) d x d y .
\end{aligned}
$$

To go further we compute

$$
-\operatorname{div}\left(y^{1-2 s} \nabla F\right)=y^{1-2 s} \sum_{j=1}^{m}\left(-\Delta\left(f_{j} \phi_{j}\right)-(1-2 s) y^{-1} \partial_{y}\left(f_{j} \phi_{j}\right)\right)=\sum_{j=1}^{m} g_{j} \phi_{j},
$$

where

$$
\begin{aligned}
g_{j} & =-\Delta f_{j}-(1-2 s) y^{-1} \partial_{y} f_{j}+\mu\left|x_{j}\right|^{-2} f_{j} \\
& =|\zeta|^{-3}\left|x_{j}\right|^{-1}\left((n+1-2 s)\left|x_{j}\right|^{2}+(\mu-k+1)|\zeta|^{2}\right) .
\end{aligned}
$$

Now (3.5) gives

$$
\int_{0}^{\infty} \int_{\mathbb{R}^{n}} y^{1-2 s}\left(|\nabla \widetilde{w}|^{2}-|\nabla w|^{2}\right) d x d y \leq \int_{0}^{\infty} \int_{\mathbb{R}^{n}} y^{1-2 s}|w|^{2}\left(\sum_{j=1}^{m} f_{j} g_{j}\right) d x d y
$$

Since

$$
\sum_{j=1}^{m} f_{j} g_{j}=\sum_{j=1}^{m}\left((n+1-2 s)\left|x_{j}\right|^{2}+(\mu-k+1)|\zeta|^{2}\right)|\zeta|^{-4} \leq(m \mu+m+1-2 s)|\zeta|^{-2},
$$

we readily obtain (3.6).

By Lemma 2.1, inequality (3.6) gives $\widetilde{w} \in \mathcal{W}^{s}$, thus $\widetilde{u}=\widetilde{w}(\cdot, 0) \in \mathcal{D}^{s}$. To conclude the proof, we compare the left-hand side of (3.6) with (2.1) (with $\widetilde{u}$ instead of $u$ ) and (2.2), and estimate the right-hand side by (2.4). 
In case $m=1$ (hence, $k=n$ ), we have the following immediate corollary

Corollary 3.2 Assume $n \geq 2$ and let $u \in \mathcal{D}^{s}$ be radially symmetric. Let $\varphi_{\mu} \in H^{1}\left(\mathbb{S}^{n-1}\right)$ be a nonconstant eigenfunction of the Laplace-Beltrami operator on $\mathbb{S}^{n-1}$ relative to the eigenvalue $\mu>0$ and satisfying (1.1). Then the function $u \varphi_{\mu} \in \mathcal{D}^{s}$ satisfies (1.3), where $c_{\mu}$ does not depend on $u$.

\section{Variational tools}

We write $\mathcal{G} \prec O(n)$ if $\mathcal{G}$ is a closed subgroup of the orthogonal group in $\mathbb{R}^{n}$ and put

$$
\mathcal{D}_{\mathcal{G}}^{s}=\left\{u \in \mathcal{D}^{s} \mid u \circ G \equiv u \text { for any } G \in \mathcal{G}\right\}, \quad S_{q}^{\mathcal{G}, \lambda}=\inf _{\substack{u \in \mathcal{D}_{\mathcal{G}}^{s} \\ u \neq 0}} J_{\lambda}(u)
$$

One finds the larger space $\mathcal{D}^{s}$ and the smallest constant $S_{q}^{\lambda}$ by choosing the trivial group $\mathcal{G}$. The space $\mathcal{D}_{\text {rad }}^{s}$ of radial functions in $\mathcal{D}^{s}$ and the infimum $S_{q}^{\mathrm{rad}, \lambda}$ are recovered by taking $\mathcal{G}=O(n)$. Trivially, one has $\mathcal{D}_{\text {rad }}^{s} \subseteq \mathcal{D}_{\mathcal{G}}^{s}$ and $S_{q}^{\lambda} \leq S_{q}^{\mathcal{G}, \lambda} \leq S_{q}^{\mathrm{rad}, \lambda}$, for any $\mathcal{G} \prec O(n)$.

Remark 4.1 Notice that $u \in \mathcal{D}_{\mathcal{G}}^{s}$ if and only if its Caffarelli-Silvestre extension $w_{u}(\cdot, y)$ is invariant with respect to the action of the group $\mathcal{G}$, for any $y>0$.

Lemma 4.2 Assume that (1.6) is satisfied. Let $\lambda>-H_{s}$ and let $\mathcal{G} \prec O(n)$. Then the infimum $S_{q}^{\mathcal{G}, \lambda}$ is positive and attained. Moreover, if $u \in \mathcal{D}_{\mathcal{G}}^{s}$ achieves $S_{q}^{\mathcal{G}, \lambda}$ then, up to a Lagrange multiplier, $u$ is nonnegative and solves (1.5). Finally, for any $\tilde{u} \in \mathcal{D}_{\mathcal{G}}^{s}$ it holds that

$$
\begin{aligned}
(q-1) \frac{Q_{\lambda}(u)}{\left\||x|^{-b} u\right\|_{q}^{q}} \int_{\mathbb{R}^{n}}|x|^{-b q}|u|^{q-2}|\tilde{u}|^{2} d x & \leq Q_{\lambda}(\tilde{u}) \\
& +(q-2) \frac{Q_{\lambda}(u)}{\left\||x|^{-b} u\right\|_{q}^{2 q}}\left(\int_{\mathbb{R}^{n}}|x|^{-b}|u|^{q-2} u \tilde{u} d x\right)^{2}
\end{aligned}
$$

where

$$
Q_{\lambda}(u):=\left\|(-\Delta)^{\frac{s}{2}} u\right\|_{2}^{2}+\lambda\left\||x|^{-s} u\right\|_{2}^{2} .
$$

Proof. We already know that $S_{q}^{\mathcal{G}, \lambda} \geq S_{q}^{\lambda}>0$. To show that the noncompact minimization problem in (4.1) has a solution we follow the outline of the proof of Theorem 0.1 in [12], 
see also [21]. By a standard convexity argument, we only need to construct a minimizing sequence that weakly converges to a nontrivial limit.

We take a small number $\varepsilon_{0}$ such that

$$
0<\varepsilon_{0}<\frac{1}{2} S_{q}^{\mathcal{G}, \lambda}
$$

Hereafter, we denote by $B_{R} \subset \mathbb{R}^{n}$ the open ball of radius $R>0$ about the origin. Since the ratio in (1.7) is invariant with respect to the transforms $u(x) \mapsto \alpha u(\beta x)$ (with $\alpha \neq 0, \beta>0$ ) of the space $\mathcal{D}_{\mathcal{G}}^{s}$ onto itself, we can find a minimizing sequence $u_{h}$ for $S_{q}^{\mathcal{G}, \lambda}$ such that

$$
\begin{gathered}
\left\||x|^{-b} u_{h}\right\|_{q}^{q}=\left(S_{q}^{\mathcal{G}, \lambda}\right)^{\frac{q}{q-2}}, \quad Q_{\lambda}\left(u_{h}\right)=\left(S_{q}^{\mathcal{G}, \lambda}\right)^{\frac{q}{q-2}}+o(1), \\
\varepsilon_{0}^{\frac{q}{q-2}} \leq \int_{B_{2}}|x|^{-b q}\left|u_{h}\right|^{q} d x \leq\left(2 \varepsilon_{0}\right)^{\frac{q}{q-2}}
\end{gathered}
$$

and $u_{h} \rightarrow u$ weakly in $\mathcal{D}^{s}$ for some $u \in \mathcal{D}^{s}$. We only have to prove that $u \neq 0$.

We argue by contradiction. If $u_{h} \rightarrow 0$ weakly in $\mathcal{D}_{\mathcal{G}}^{s}$, we can use Rellich theorem to get that $|x|^{-b} u_{h} \rightarrow 0$ strongly in $L_{\text {loc }}^{q}\left(\mathbb{R}^{n} \backslash\{0\}\right)$. So, (4.5) implies

$$
\varepsilon_{0}^{\frac{q}{q-2}} \leq \int_{B_{1}}|x|^{-b q}\left|u_{h}\right|^{q} d x+o(1) .
$$

By Ekeland's variational principle we can assume that

$$
(-\Delta)^{s} u_{h}+\lambda|x|^{-2 s} u_{h}-|x|^{-b q}\left|u_{h}\right|^{q-2} u_{h} \rightarrow 0 \quad \text { in }\left(\mathcal{D}_{\mathcal{G}}^{s}\right)^{\prime} .
$$

Take a radial function $\varphi \in \mathcal{C}_{0}^{\infty}\left(B_{2}\right)$ such that $\varphi \equiv 1$ on $B_{1}$. Then $\varphi^{2} u_{h}$ is a bounded sequence in $\mathcal{D}_{\mathcal{G}}^{s}$. By [21, Lemma 2.1], there exists a constant $c>0$ depending on $\varphi$ but not on $h$, such that

$$
\left|\left\langle(-\Delta)^{s} \varphi u_{h}, \varphi u_{h}\right\rangle-\left\langle(-\Delta)^{s} u_{h}, \varphi^{2} u_{h}\right\rangle\right|^{2} \leq c\left\langle(-\Delta)^{s} u_{h}, u_{h}\right\rangle\left\|u_{h}\right\|_{L^{2}\left(B_{2}\right)}^{2} .
$$

In particular, thanks to Rellich theorem we obtain

$$
\left\langle(-\Delta)^{s} u_{h}, \varphi^{2} u_{h}\right\rangle=\left\langle(-\Delta)^{s}\left(\varphi u_{h}\right), \varphi u_{h}\right\rangle+o(1)=\left\|(-\Delta)^{\frac{s}{2}}\left(\varphi u_{h}\right)\right\|_{2}^{2}+o(1),
$$

that compared with the definition of $S_{q}^{\mathcal{G}, \lambda}$ leads to

$$
\left\langle(-\Delta)^{s} u_{h}, \varphi^{2} u_{h}\right\rangle+\lambda|||x|^{-s} \varphi u_{h}\left\|_{2}^{2} \geq S_{q}^{\mathcal{G}, \lambda}\right\||x|^{-b} \varphi u_{h} \|_{q}^{2}+o(1) .
$$


On the other hand, (4.7), Hölder's inequality and (4.5) give

$$
\begin{aligned}
\left\langle(-\Delta)^{s} u_{h}, \varphi^{2} u_{h}\right\rangle & +\lambda\left\||x|^{-s} \varphi u_{h}\right\|_{2}^{2}=\int_{\mathbb{R}^{n}}|x|^{-b q}\left|u_{h}\right|^{q-2}\left|\varphi u_{h}\right|^{2} d x+o(1) \\
& \leq\left(\int_{B_{2}}|x|^{-b q}\left|u_{h}\right|^{q} d x\right)^{\frac{q-2}{q}}\left\||x|^{-b} \varphi u_{h}\right\|_{q}^{2} \leq 2 \varepsilon_{0}\left\||x|^{-b} \varphi u_{h}\right\|_{q}^{2}+o(1) .
\end{aligned}
$$

Taking (4.8) into account, we see that

$$
S_{q}^{\mathcal{G}, \lambda}\left\||x|^{-b} \varphi u_{h}\right\|_{q}^{2} \leq 2 \varepsilon_{0}\left\||x|^{-b} \varphi u_{h}\right\|_{q}^{2}+o(1)
$$

which implies $\left\||x|^{-b} \varphi u_{h}\right\|_{q}=o(1)$ by (4.3). Since $\varphi \equiv 1$ on $B_{1}$, we infer from (4.6)

$$
\varepsilon_{0}^{\frac{q}{q-2}} \leq \int_{B_{1}}|x|^{-b q}\left|u_{h}\right|^{q} d x+o(1) \leq \int_{\mathbb{R}^{n}}|x|^{-b q}\left|\varphi u_{h}\right|^{q} d x+o(1)=o(1),
$$

a contradiction. Therefore, $S_{q}^{\mathcal{G}, \lambda}$ is achieved by some function $u \in \mathcal{D}_{\mathcal{G}}^{s}$.

Since $Q_{\lambda}(v)>Q_{\lambda}(|v|)$ for sign-changing function $v \in \mathcal{D}^{s}$, see [19, Theorem 6], we can assume that $u$ is nonnegative.

Next, for any $G \in O(n)$ we have $J_{\lambda}(u \circ G)=J_{\lambda}(u)$. As a consequence of the Principle of symmetric criticality [25] we have that $\mathcal{D}_{\mathcal{G}}^{s}$ is a natural constraint for $J_{\lambda}$, and thus $u$ is a critical point for $J_{\lambda}$ on the whole $\mathcal{D}^{s}$. So, $u$ solves the fractional differential equation in (1.5), up to a Lagrange multiplier.

Finally, the proof of (4.2) is a simple computation, based on the fact that the function $f(t)=J_{\lambda}(u+t \widetilde{u})$ attains its minimum value at $t=0$, hence $f^{\prime}(0)=0, f^{\prime \prime}(0) \geq 0$.

By taking $\mathcal{G}=\left\{\operatorname{Id}_{\mathbb{R}^{n}}\right\}$ and then $\mathcal{G}=O(n)$ in Lemma 4.2, we immediately obtain the next existence result.

Corollary 4.3 Assume that (1.6) is satisfied. If $\lambda>-H_{s}$, then the infimum $S_{q}^{\lambda}$ in (1.7) and the infimum

$$
S_{q}^{r a d, \lambda}=\inf _{\substack{u \in \mathcal{D}^{s} \\ \text { rad } \\ u \neq 0}} J_{\lambda}(u)
$$

are attained by nonnegative solutions to (1.5).

Remark 4.4 Clearly Lemma 4.2 and Corollary 4.3 hold also in case $n=1,0<s<\frac{1}{2}$, with no changes in the proof. 
In general, solutions achieving the infima $S_{q}^{\mathcal{G}, \lambda}$ for different groups $\mathcal{G} \prec O(n)$ can coincide. To obtain distinct solutions, we will use a special class of groups in $O(n)$.

We need to introduce some notation. Recall that $n=k m$. To any rotation $G \in O(k)$ and any permutation $P \in S_{m}$ we associate the rotation

$$
P G \in O(n):\left(x_{1}, \ldots, x_{m}\right) \mapsto P\left(G x_{1}, \ldots G x_{m}\right), \quad x_{j} \in \mathbb{R}_{j}^{k}
$$

Further, to any subgroup $\mathcal{G}_{k} \prec O(k)$ we associate the following group of rotations in $\mathbb{R}^{n}$,

$$
\widetilde{\mathcal{G}}_{k}=\left\{P G \mid P \in S_{m}, \quad G \in \mathcal{G}_{k}\right\} \prec O(n) .
$$

Let $u \in \mathcal{D}_{\widetilde{\mathcal{G}}_{k}}^{s}$. Then $u$ is $\mathcal{G}_{k}$-invariant in each variable $x_{j}$ and is invariant with respect to any permutation of the $m$-tuple of vectors $\left(x_{1}, \ldots, x_{m}\right)$.

We will say that functions in $\mathcal{D}_{\widetilde{O}(k)}^{s}$ are $k$-radially symmetric (in [23] they are called $(k, 0)$-radial, see also [17, 24]). Notice that a $k$-radially symmetric function depends only on $\left|x_{1}\right|, \ldots,\left|x_{m}\right|$ and is invariant with respect to permutations of its variables.

Clearly, $n$-radially symmetric functions are radial.

Lemma 4.5 (Basic Lemma) Let $n=k m$ with $k \geq 2$ and $m \geq 1$, and let $\mathcal{G}_{k}$ be a proper closed subgroup of $O(k)$. If $\lambda>0$ is large enough, then no extremal for $S_{q}^{\widetilde{\mathcal{G}}_{k}, \lambda}$ can be $k$-radially symmetric.

Proof. Let $u \in \mathcal{D}_{\widetilde{\mathcal{G}}_{k}}^{s}$ be a $k$-radially symmetric function achieving the best constant $S_{q}^{\widetilde{\mathcal{G}}_{k}, \lambda}$ for some $\lambda>0$. We take $\widetilde{u}$ as in Lemma 3.1, where $\phi$ is given by (3.1) while $\mu$ is defined in (3.2). Clearly $\widetilde{u} \in \mathcal{D}_{\widetilde{\mathcal{G}}_{k}}^{s}$, so that formula (4.2) in Lemma 4.2 applies.

Using (3.3) we can rewrite (4.2) as follows:

$$
(q-1) Q_{\lambda}(u) \leq Q_{\lambda}(\widetilde{u}) \leq Q_{\lambda}(u)+c_{\mu} \int_{\mathbb{R}^{n}}|x|^{-2 s}|u|^{2} d x
$$

(the last inequality follows by (3.4) $)$, i.e.

$$
(q-2) Q_{\lambda}(u) \leq c_{\mu} \int_{\mathbb{R}^{n}}|x|^{-2 s}|u|^{2} d x .
$$

Now we use the Hardy inequality to estimate $Q_{\lambda}(u)>\left(H_{s}+\lambda\right)\left\||x|^{-s} u\right\|_{2}^{2}$. We infer that $(q-2)\left(H_{s}+\lambda\right)<c_{\mu}$. We proved that if $\lambda \geq-H_{s}+\frac{c_{\mu}}{q-2}$ then a $k$-radially symmetric function cannot provide the constant $S_{q}^{\widetilde{\mathcal{G}}}, \lambda$. 


\section{Proofs of the main results}

Proof of Theorem 1.1. This is an immediate consequence of Lemma 4.5, with $m=1$, $k=n$ (so that $k$-radially symmetric functions are radially symmetric) and $\mathcal{G}=\left\{\operatorname{Id}_{\mathbb{R}^{n}}\right\}$.

Proof of Theorem 1.2. We identify $\mathbb{R}^{n}$ with $\left(\mathbb{R}^{2}\right)^{m}, m \geq 1$ and introduce the polar coordinates $x_{j}=\left(r_{j}, \sigma_{j}\right)$ for points in $\mathbb{R}^{2}$.

Following [23], for any integer $t>1$ we denote by $\mathcal{G}_{2}^{t} \prec O(2)$ the group generated by a

rotation of $\frac{2 \pi}{t}$ and by $\widetilde{\mathcal{G}}_{2}^{t}$ the corresponding subgroup of $O(n)$, see (4.9), Then we denote by $u_{t}$ the nonnegative solution to (1.5) solving the minimization problem

$$
J_{\lambda}\left(u_{t}\right)=\min _{u \in \mathcal{D}_{\widetilde{\mathcal{G}}_{2}^{t}}^{s}} J_{\lambda}(u)
$$

compare with Lemma 4.2. We prove that for any pair of distinct integers $t, T$, the functions $u_{T}, u_{t}$ cannot coincide up to rotations, provided that $\lambda$ is large enough.

First, we face the case when $T=h t, h>1$, is a multiple of the integer $t$. Recall Lemma 4.5 and find $\lambda>0$ large, so that $u_{T}$ is not $k$-radially symmetric. We introduce the function

$$
v_{t}\left(\left(r_{1}, \sigma_{1}\right), \ldots,\left(r_{m}, \sigma_{m}\right)\right)=u_{T}\left(\left(r_{1}, \frac{1}{h} \sigma_{1}\right), \ldots,\left(r_{m}, \frac{1}{h} \sigma_{m}\right)\right) .
$$

Easily, $v_{t} \in \mathcal{D}_{\widetilde{\mathcal{G}}_{2}^{t}}^{s}$ and

$$
\int_{\mathbb{R}^{n}}|x|^{-2 s}\left|v_{t}\right|^{2} d x=\int_{\mathbb{R}^{n}}|x|^{-2 s}\left|u_{T}\right|^{2} d x, \quad \int_{\mathbb{R}^{n}}|x|^{-b q}\left|v_{t}\right|^{q} d x=\int_{\mathbb{R}^{n}}|x|^{-b q}\left|u_{T}\right|^{q} d x .
$$

Let $w_{T}=w_{u_{T}}$ be the Caffarelli-Silvestre extension of $u_{T}$ and consider the function

$$
\widetilde{w}_{t}\left(\left(r_{1}, \sigma_{1}\right), \ldots,\left(r_{m}, \sigma_{m}\right), y\right)=w_{T}\left(\left(r_{1}, \frac{1}{h} \sigma_{1}\right), \ldots,\left(r_{m}, \frac{1}{h} \sigma_{m}\right), y\right) .
$$

We have $\widetilde{w}_{t}(x, 0)=v_{t}(x)$. Moreover, from the formula

$$
|\nabla w|^{2}=\sum_{j=1}^{m}\left(\left|\partial_{r_{j}} w\right|^{2}+\frac{1}{r_{j}^{2}}\left|\partial_{\sigma_{j}} w\right|^{2}\right)+\left|\partial_{y} w\right|^{2}
$$

and taking into account the relation $\partial_{\sigma_{j}} \widetilde{w}_{t}=\frac{1}{h} \partial_{\sigma_{j}} w_{T}$, we get

$$
\int_{0}^{\infty} \int_{\mathbb{R}^{n}} y^{1-2 s}\left|\nabla \widetilde{w}_{t}\right|^{2} d x d y<\int_{0}^{\infty} \int_{\mathbb{R}^{n}} y^{1-2 s}\left|\nabla w_{T}\right|^{2} d x d y
$$


because $w_{T}(\cdot, y)$ cannot be $k$-radially symmetric, and thus $\partial_{\sigma_{j}} w_{T} \not \equiv 0$.

Formulae (2.1) with $\widetilde{u}_{t}$ instead of $u$, (5.1) and (2.2) give

$$
\begin{aligned}
\int_{\mathbb{R}^{n}}\left|(-\Delta)^{\frac{s}{2}} v_{t}\right|^{2} d x & \leq C_{s} \int_{0}^{\infty} \int_{\mathbb{R}^{n}} y^{1-2 s}\left|\nabla \widetilde{w}_{t}\right|^{2} d x d y \\
& <C_{s} \int_{0}^{\infty} \int_{\mathbb{R}^{n}} y^{1-2 s}\left|\nabla w_{T}\right|^{2} d x d y=\int_{\mathbb{R}^{n}}\left|(-\Delta)^{\frac{s}{2}} u_{T}\right|^{2} d x
\end{aligned}
$$

and we infer that

$$
J_{\lambda}\left(u_{t}\right) \leq J_{\lambda}\left(v_{t}\right)<J_{\lambda}\left(u_{T}\right)
$$

for $\lambda$ large enough. Thus, the statement is proved for $T=h t$.

In the case of general distinct intergers $T, t$ we define $\hat{t}$ as the least common multiple of the pair $T$, $t$. If $u_{T}=u_{t} \circ G$ for some rotation $G \in O(n)$ then $u_{t}$ is $\widetilde{\mathcal{G}}_{2}^{\hat{t}}$-invariant. But this implies $J_{\lambda}\left(u_{\hat{t}}\right) \leq J_{\lambda}\left(u_{t}\right)=J_{\lambda}\left(u_{T}\right)$, that is impossible for $\lambda$ large enough by (5.2). The proof is complete.

Before the proof of Theorem 1.3, we make a remark.

Remark 5.1 Notice that if $n$ has $\ell$ distinct divisors then it is easy to obtain $\ell$ distinct solutions for $\lambda$ large enough. Let $1<k_{j}<n, j=1, \ldots, \ell-2$, be the distinct nontrivial divisors of $n$, so that $\mathbb{R}^{n}=\left(\mathbb{R}^{k_{j}}\right)^{\frac{n}{k_{j}}}$. Thanks to Lemmata 4.2 and 4.5, for $\lambda$ large the $\ell$ solutions achieving the best constants $S_{q}^{\mathcal{G}, \lambda}$ for $\mathcal{G}=\{I d\}, \mathcal{G}=\widetilde{O}\left(k_{j}\right)$ and $\mathcal{G}=O(n)$ are distinct modulo rotations.

To manage a general case, including prime dimensions, some extra argument is needed.

Proof of Theorem 1.3. Take any $u \in \mathcal{D}^{s}$ and its Caffarelli-Silvestre extension $w_{u}$. We denote by $u^{*}$ and $w^{*}(\cdot, y)$ the symmetrization along spheres of $u$ and $w_{u}(\cdot, y)$, respectively. By [16, Theorem 2.31], such symmetrization diminishes the $L^{2}\left(\mathbb{R}^{n}\right)$-norm of $\nabla_{x} w$. Therefore we get

$$
\int_{0}^{\infty} \int_{\mathbb{R}^{n}} y^{1-2 s}\left|\nabla w^{*}\right|^{2} d x d y \leq \int_{0}^{\infty} \int_{\mathbb{R}^{n}} y^{1-2 s}\left|\nabla w_{u}\right|^{2} d x d y .
$$

Since evidently the spherical symmetrization keeps weighted norms,

$$
\int_{\mathbb{R}^{n}}|x|^{-2 s}\left|u^{*}\right|^{2} d x=\int_{\mathbb{R}^{n}}|x|^{-2 s}|u|^{2} d x, \quad \int_{\mathbb{R}^{n}}|x|^{-b q}\left|u^{*}\right|^{q} d x=\int_{\mathbb{R}^{n}}|x|^{-b q}|u|^{q} d x,
$$


formulae (2.1) and (2.2) imply $J_{\lambda}\left(u^{*}\right) \leq J_{\lambda}(u)$. Therefore, if $U$ achieves $S_{q}^{\lambda}$ then it is axisymmetric, i.e., up to rotations, $U(x)=U(|x|, \vartheta)$ where $\vartheta=\cos ^{-1}\left(x_{n} /|x|\right)$ is the angle between $x$ and the axis $O x_{n}$. Moreover, if $U$ is not radial (that holds for $\lambda$ large enough) then it is strictly monotone with respect to $\vartheta$.

Now we define four groups such that corresponding minimizers are different for $\lambda$ large enough in arbitrary dimension. Besides full group $O(n)$ and the trivial group, they are $O(n-1) \times \mathbb{Z}_{2}$ (modulo rotations, corresponding functions depending only on $|x|$ and $|\cos (\vartheta)|)$ and the symmetry group of the right simplex.

By Lemma 4.5, minimizers corresponding to the last three groups cannot be radial for $\lambda$ large enough. Since the global minimizer is axisymmetric and monotone with respect to $\vartheta$, it cannot be invariant with respect to the last two groups. Similarly, the minimizer generated by $O(n-1) \times \mathbb{Z}_{2}$ is monotone with respect to $\vartheta$ in both half-spaces, and so it cannot be invariant with respect to the simplex group.

\section{Conclusions}

We furnished a powerful tool that can be applied to a large class of variational equations driven by the fractional Laplace operator $(-\Delta)^{s}$ of order $s \in(0,1)$. One of the main steps is Lemma 3.1, that allows to compare the $L^{2}$ norms of $(-\Delta)^{\frac{s}{2}} u$ and $(-\Delta)^{\frac{s}{2}}\left(u \varphi_{\mu}\right)$, where $u$ is a given function of $k$ variables $x_{j} \in \mathbb{R}^{m}$ depending only on $\left|x_{j}\right|$, and $\varphi_{\mu}$ is a suitably normalized eigenfunction of the Laplace-Beltrami operator on the unit sphere in $\mathbb{R}^{m k}$.

Then we take as model a nonlocal variational equation related to the fractional CaffarelliKohn-Nirenberg inequality to illustrate how Lemma 3.1 can be used in order to obtain symmetry breaking and multiplicity phenomena.

Differently from the local case $s=1$, an efficient Emden-Fowler transform is not available and positive radial solutions to (1.5) are not explicitly known; their uniqueness (up to dilations) is an open question as well, that makes the problem more challenging.

\section{Acknowledgements}

R.M. was partially supported by PRID project VARPROGE.

This work does not have any conflicts of interest 


\section{References}

[1] M. Bhakta and R. Musina, Entire solutions for a class of variational problems involving the biharmonic operator and Rellich potentials, Nonlinear Anal. 75 (2012), no. 9, 3836-3848.

[2] L. Caffarelli and L. Silvestre, An extension problem related to the fractional Laplacian, Comm. Part. Diff. Eqs. 32 (2007), no. 7-9, 1245-1260.

[3] F. Catrina and Z.-Q. Wang, On the Caffarelli-Kohn-Nirenberg inequalities: sharp constants, existence (and nonexistence), and symmetry of extremal functions, Comm. Pure Appl. Math. 54 (2001), no. 2, 229-258.

[4] P. Caldiroli and G. Cora, Entire solutions for a class of fourth-order semilinear elliptic equations with weights, Mediterr. J. Math. 13 (2016), no. 2, 657-675.

[5] P. Caldiroli and R. Musina, Symmetry breaking of extremals for the Caffarelli-Kohn-Nirenberg inequalities in a non-Hilbertian setting, Milan J. Math. 81 (2013), no. 2, 421-430.

[6] A. Cotsiolis and N. K. Tavoularis, Best constants for Sobolev inequalities for higher order fractional derivatives, J. Math. Anal. Appl. 295 (2004), no. 1, 225-236.

[7] S. Dipierro, L. Montoro, I. Peral and B. Sciunzi, Qualitative properties of positive solutions to nonlocal critical problems involving the Hardy-Leray potential, Calc. Var. Partial Differential Equations 55 (2016), no. 4, Art. 99, 29 pp.

[8] J. Dolbeault, M. J. Esteban and M. Loss, Rigidity versus symmetry breaking via nonlinear flows on cylinders and Euclidean spaces, Invent. Math. 206 (2016), no. 2, 397-440.

[9] J. Dolbeault, M. J. Esteban and G. Tarantello, The role of Onofri type inequalities in the symmetry properties of extremals for Caffarelli-Kohn-Nirenberg inequalities, in two space dimensions, Ann. Sc. Norm. Super. Pisa Cl. Sci. (5) 7 (2008), no. 2, 313-341.

[10] B. Dyda, A. Kuznetsov and M. Kwaśnicki, Fractional Laplace operator and Meijer G-function, Constr. Approx. 45 (2017), no. 3, 427-448.

[11] V. Felli and M. Schneider, Perturbation results of critical elliptic equations of Caffarelli-KohnNirenberg type, J. Differential Equations 191 (2003), no. 1, 121-142.

[12] M. Gazzini and R. Musina, On a Sobolev-type inequality related to the weighted $p$-Laplace operator, J. Math. Anal. Appl. 352 (2009), no. 1, 99-111.

[13] M. Gazzini and R. Musina, Hardy-Sobolev-Maz'ya inequalities: symmetry and breaking symmetry of extremal functions, Commun. Contemp. Math. 11 (2009), no. 6, 993-1007.

[14] L. Grafakos and G. Teschl, On Fourier transforms of radial functions and distributions, J. Fourier Anal. Appl. 19 (2013), no. 1, 167-179.

[15] I. W. Herbst, Spectral theory of the operator $\left(p^{2}+m^{2}\right)^{1 / 2}-Z e^{2} / r$, Comm. Math. Phys. 53 (1977), no. 3, 285-294.

[16] B. Kawohl, Rearrangements and convexity of level sets in PDE, Lecture Notes in Mathematics, 1150, Springer-Verlag, Berlin, 1985. 
[17] S. B. Kolonitskii and A. I. Nazarov, Multiplicity of solutions to the Dirichlet problem for generalized Hénon equation, Probl. Mat. Analiza 35 (2007), 91-110 (Russian); English transl.: J. Math. Sci. (N.Y.) 144 (2007), no. 6, 4624-4644.

[18] R. Musina, Partial differential equations - existence and multiplicity results for a weighted $p$-Laplace equation involving Hardy potentials and critical nonlinearities, Atti Accad. Naz. Lincei Rend. Lincei Mat. Appl. 20 (2009), no. 2, 127-143.

[19] R. Musina and A. I. Nazarov, On the Sobolev and Hardy constants for the fractional Navier Laplacian, Nonlinear Anal. 121 (2015), 123-129.

[20] R. Musina and A. I. Nazarov, On fractional Laplacians 2, Ann. Inst. H. Poincaré Anal. Non Linéaire 33 (2016), no. 6, 1667-1673.

[21] R. Musina and A. I. Nazarov, Fractional Hardy-Sobolev inequalities on half spaces, Nonlinear Anal. 178 (2019), 32-40.

[22] R. Musina and A. I. Nazarov, A weighted estimate for generalized harmonic extensions, preprint arXiv:1903.02649 (2019).

[23] A. I. Nazarov, On solutions of the Dirichlet problem for an equation involving the $p$-Laplacian in a spherical layer, Proc. St.Petersburg Math. Soc. 10 (2004), 33-62 (Russian); English transl.: AMS Transl. Series 2, 214 (2005), 29-57.

[24] A. I. Nazarov and B. O. Neterebskiı̌, Multiplicity of positive solutions of a quasilinear equation generated by the Il'in-Caffarelli-Kohn-Nirenberg inequality, ZNS POMI, 444 (2016), 98-109 (Russian); English transl.: J. Math. Sci. (N.Y.) 224 (2017), N3, 448-455.

[25] R. S. Palais, The principle of symmetric criticality, Comm. Math. Phys. 69 (1979), no. 1, 19-30.

[26] D. Smets, M. Willem and J. Su, Non-radial ground states for the Hénon equation, Commun. Contemp. Math. 4 (2002), no. 3, 467-480.

[27] A. P. Scheglova, Multiplicity of solutions to a boundary-value problem with nonlinear Neumann condition, Probl. Mat. Analiza, 30 (2005), 121-144 (Russian); English transl.: J. Math. Sci. (N.Y.) 128 (2005), no. 5, 3306-3333.

[28] N. S. Ustinov, Multiplicity of Positive Solutions to the Boundary-Value Problems for Fractional Laplacians, ZNS POMI, 459 (2017), 104-126 (Russian); English transl.: J. Math. Sci. (N.Y.) 236 (2019), no. 4, 446-460. 